\title{
EMCV: Enhancing the Security of the Mobile Commerce using Voice Features
}

\author{
Sonal Gupta \\ Lovely Professional University, \\ CSE Deptt., Chaheru, 144402, \\ Kapurthala, Punjab,India
}

\begin{abstract}
M-Commerce is an emerging market. As with any emerging market there are some significant opportunities and risks .In M-Commerce security risk is major risk which this proposed system will try to remove. The most important element of MCommerce is security issues and how this proposed system can make it safe for customers to feel comfortable while using mobile phones. Biometric authentication technology is best to resolve security issues related to M-Commerce as this will provide one's uniqueness. In the proposed system MCommerce Web Page is created in PHP which will then integrate with Matlab. Start by taking input speech through microphone whose voice need to be authenticated. Then features will be extract from voice through combined use of algorithms Linear Prediction Coding (LPC), and MelFrequency Cepstrum Coefficients (MFCC) to increase reliability and recognition quality. After feature extraction, matching of those features will be done through both algorithms Hidden Markov Modeling (HMM) and Vector Quantization (VQ) separately. At most one feature should be matched from both algorithms separately then only authenticated person will get login through Web Page.
\end{abstract}

\section{General Terms}

Biometric authentication, Voice Recognition

\section{Keywords}

M-Commerce,PHP, Mel-Frequency Cepstrum

Coefficients, Hidden Markov Modeling, Vector

Quantization.,SQLServer,JDBC Driver

\section{INTRODUCTION}

The wide availability of wireless communication and mobile devices emerged a new form of business. Emergence of MCommerce changed the way businesses managed and created traditionally. M-Commerce has not only changed traditional business but also brought cultural and social transformations within nation. Basically, M-Commerce is usage of handheld wireless devices to interact, communicate and make transactions over high speed connection to the Internet.As phone technology is advancing day by day,consumers of wireless devices can access services and content anytime and anywhere. For example, They will be able to use mobile phones to access bank accounts ,ticket reservations and receive special promotions and orders can be generate anytime. M-Commerce websites provides a capability to commit transactions anytime and anywhere using mobile devices with extensive Internet accessibility.The proposed system try to resolve the problem faced when we are dealing with M-Commerce. This important element is security issues that how customers safely perform their work and feel comfortable when using mobile phones only after ensuring the quality of the security level.

There are many existing techniques available like Regular WEP, Account Lock out, Biometric Identification. This proposed system is based on Biometric authentication. Every technique has some advantages and disadvantages as well. Reasons of choosing Biometric authentication as this method really authenticate user by taking biological characteristics in form of input to system. These biological characteristics are permanent(more or less), unchangeable and do not pass to other users as easily as users pass their passwords and cards to others. Customers are free from worry of being stolen by someone like keys and cards. Passwords may forget sometime where biometric characteristics need not to be remember. For example, In order to receive benefits. There are some cases where person can claim multiple identities by showing fraudulent documents where biometric identification is free from such fraudulent activities. In fact it give contribution in fraud detection.

Biometric Identification provides authentication service which is concerned that is the person is same whom he is claiming to be. Nowadays it is easy to crack passwords of someone by hackers. Moreover, there are so many software's available in market which will allow novice hackers to get access to other account by cracking their passwords. In result of this, one can lose their personal and valuable information just because of lack of security. Therefore Authentication is an important feature of any secure web site. Every time a client browses to a web site, it needs to be authenticated before it can access the resources it is requesting. Biometric authentication technology is best to resolve security issues related to $\mathrm{M}$-Commerce These biometric data identify individual person uniquely preferably with inexpensive equipment, with an immediate result as in case of voice authentication which is cheap method to identify person the one who claims it to be. This Biometric authentication includes our fingerprint, facial analysis, voice authentication, retinal scans. In this the proposed system work on voice authentication to resolve security issues as this is unique from person to person. It also proves to be a huge benefit to those with limited mobility or some other kind of disability that makes it difficult to use a keyboard and mouse. People with disability do not authenticate themselves by using keyboard and mouse. Alone voice recognition will help them to authenticate themselves without touching keyboard or mouse. Handicap people has to give their biometric data by just speaking out any word in same tone so that at least system will collect as many samples 
as need for correct authentication. Then matching will be done against their previously stored samples in database. If matched then handicap people will get access to site. In case if samples does not match with previously stored one then System will prompt user to give their biometric data that is voice sampling again. Voice recognition will authenticate person whom he claims to be.

\section{VOICE RECOGNITION}

Voice authentication is matching features of speech with previously stored speech samples that is voiceprint. Then we can find which speech resembles to that recorded speech therefore in this way system can identify individual's voice.

\section{APPROACH FOR DEVELOPING THE PROPOSED SYSTEM}

The proposed system describes the security model on voice authentication which comprised of various modules. Each module uses different techniques and algorithms to perform its specific tasks. After a particular module completes its task, its output will become input for the next module. In the end the combined effort of each module will be displayed.

\subsection{User Registration:-}

3.1.1 Construct M-Commerce Web Page using PHP as it easily embeds in HTML and free of cost . Using XHTML, CSS to make web pages mobile will help in displaying web page on screens of different sizes and also easily navigate from one browser to another in mobile screen. In Web page there will be three buttons Register, Login, Visit the site along with one textbox where user will enter his/her valid username during registration time which will compare against username which user will enter during login time.Integrate Web Page with Matlab. Make Connection between SQLServer and MATLAB using conn=database('datasourcename',","). As shown in figure 3 .

3.1.2 If user has not been registered before then user will click on Register button . On Clicking Registration Button System will prompt user to enter username first. After getting username from user ,System will ask for voice sampling and this input will given by user using microphone so as to minimize noise. System will record at least ten samples from user so to achieve accuracy in feature extraction. These recording of voice will done using wavrecord(n,Fs) which records $n$ samples of an audio signal, sampled at rate of Fs $\mathrm{Hz}$ (samples per second). After recording wave signal write this wave in any created file using Wavwrite (y,filename) .This function writes the data stored in the variable $y$ to a WAVE file called filename. Double Click on variable y in WorkSpace which is on left side of Command Window in Matlab. Variable Editor will open containing multiple values in single column. Store these values in voice database.

3.1.3 Data will send from voice database to features database where features will extract by combined use of MFCC (MelFrequency Cepstrum Coefficients) and LPC (Linear Prediction Coding) so that maximum features will be extracted and also it will improve recognition quality and its reliability. Features will save in feature database and message will send to user for successful registration. As shown in figure 1.

\subsection{User Login:-}

3.2.1 User click on Login button and username database will ask for username. After getting username, username database will match it against username previously stored in it. If matched then username send to voice database which will further ask for voice otherwise username database will prompt user again for username if not matched. User's voice will be recorded and save in voice database .Voice will be send to feature database in which combined use of algorithm MFCC and LPC is used to extract features from it .

3.2.2 Features of user which were previously saved in feature database during registration time will be compared against features extracted during login time through HMM (Hidden Markov Model) and VQ(Vector Quantization) separately. This will be checked in featured matched database that if at least single feature from both algorithm will match then only user will be authenticated and user will successfully login and get access to M-Commerce website. As shown in figure 2

\subsubsection{Mobile simulation is done.}

\subsection{USER Visit Page:-}

User will just visit Web Page as anonymous user who will visit web pages and will not authenticate to access any resources without login.

\section{4 .PROPOSED ALGORITHM}

The outlined algorithm for the system looks like following set of steps. The coordination between these steps must be there because they follow a particular order

1)Call Construct page module written in PHP and next module will not come until user enter his/her username or does not click on any of the button .

2) Call the recording of voice module by using waverecord and wavewrite function. Repeat this step until user does not complete their number of samplings.

3) Call the Feature Extraction module and Extract the Useful features from the sampled voice recorded by given user. Perform this step only once. This module will also extract features of voice when user will click on Login.

4) Call Feature Comparison module. Perform this step until the entire features of voice stored during registration click will compare with features of voice extracted during Login Click.

5) Call the Verification done module to verify the authenticity of user.

Algorithm Ends here 


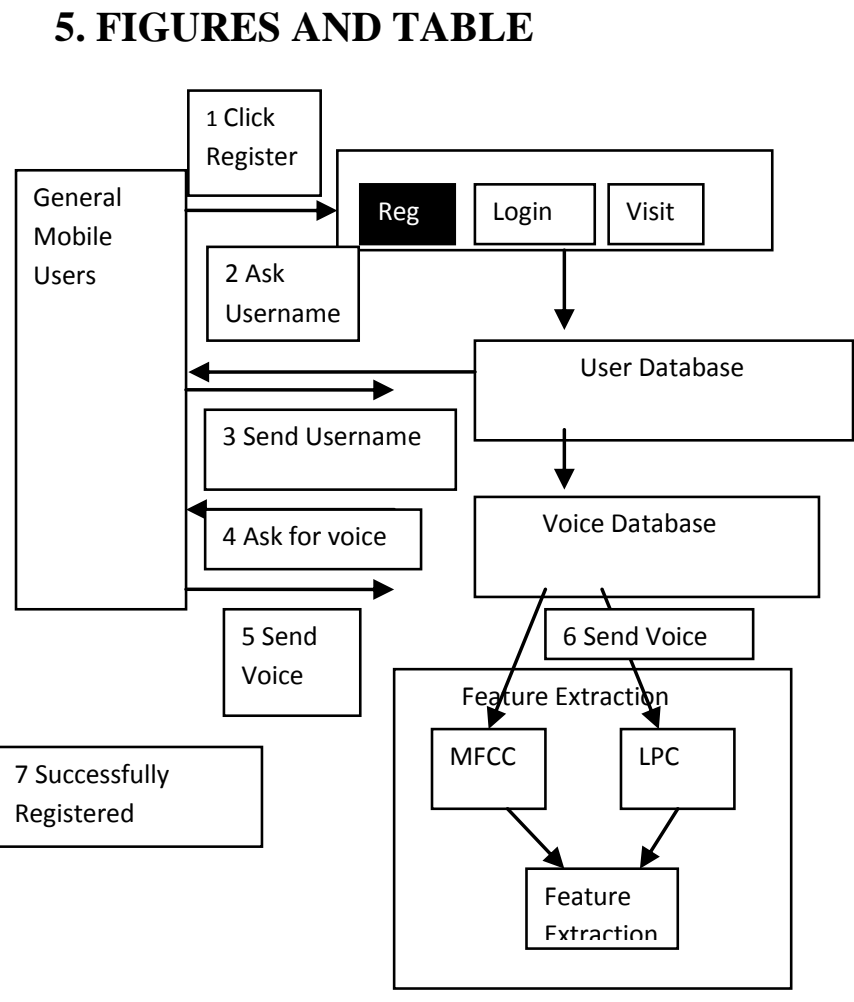

Figure1: Process flow of security model M-Commerce Model when user clicks on register button

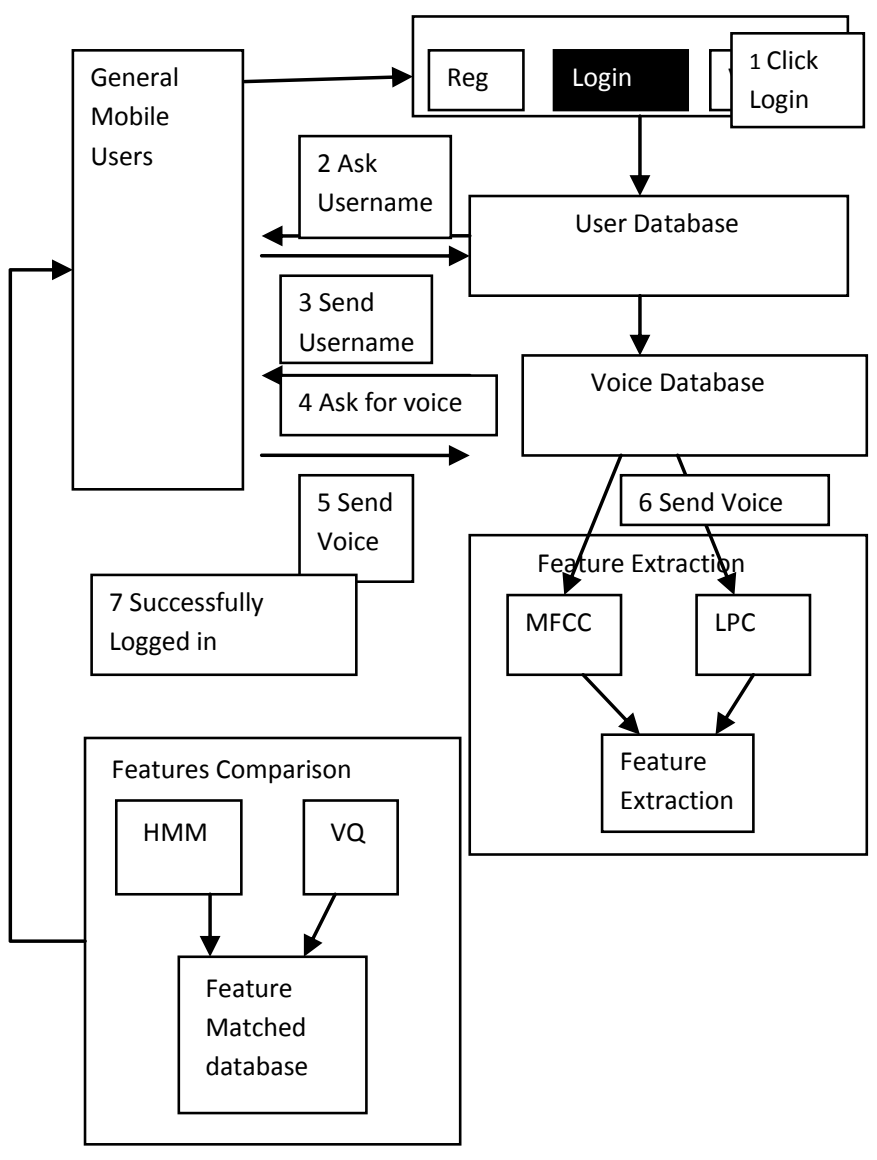

Figure2: Process flow of security model M-Commerce Model when user clicks on Login button

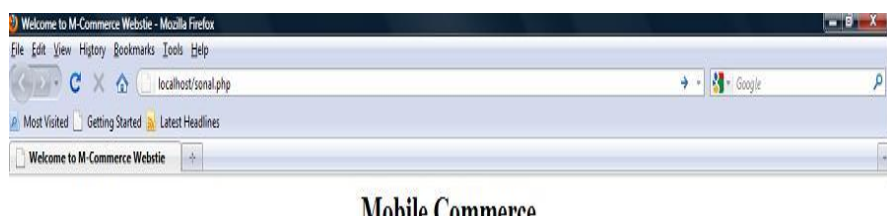

Mobile Commerce

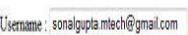

Figure 3: M-commerce Web Page

\section{PURPOSE OF THE RESEARCH}

The Purpose of proposed system of doing this research is to prepare such a system which will resolve security issues and enhance security of M-Commerce. There are many security issues which result in loss of valuable information. If security issues have been resolved then many people will attract and will do transactions through mobile .Moreover these proposed system will also efficiently use the various available Speech Recognition algorithms in voice authentication. The availability of various algorithms in computer vision field has made it possible for the 
programmers to construct a system which will authenticate every user before they login in site. This authentication will be done by first taking speech input through microphone then it will be represented in frequency time plane then acoustic features will be extracted from signal which will be input to the features comparison. If features matched then given speaker input is legal or not. Sometimes person voice may change little due to sore throat or emotions so this proposed system will also help in tackling these types of problems. The Proposed System will also support handicap people to get access to sites being authenticated through voice without touching keyboard or mouse. It will also identified several acoustic features in the voices of people recorded so that through voice one can identify one's mental situation whether speaker is in depression, at high risk of suicide so it has wider scope.

\section{7 .BENEFITS OF THE RESEARCH}

With the introduction of advanced phone technology, consumers of mobile devices will be able to access content and services anytime, anywhere. Nowadays, M-Commerce is an emerging market as with any emerging market there are significant opportunities and significant risks and major risk is security risk which this proposed system will try to remove. If no proper security is provided then users may lose their valuable information so this system will help in enhancing security which will be useful for large corporations to do their transactions safely. Moreover, System used for transactions has to absolutely secure otherwise business will lose customers if the mobile security system is not secure enough. Authentication service is basically concerned that is the person is same whom he is claiming to be and prevent illegal users to access resources.

If different biometric technology will be compared then Voice recognition technology on the user's mobile phone doesn't need to attach other devices and it also has more advantage over other in case of remote users. Voice recognition includes some more benefits like ease of use, willing participation of subject is not required by user and uses of legacy data. Authentication through voice is one of the cheapest method to authenticate individual in terms of cost.

\section{CONCLUSIONS}

This proposed system will resolve security issues and enhance security of M-Commerce which results in increasing mobile users. With the introduction of advanced phone technology with proposed system, consumers of mobile devices will be able to access content and services anytime, anywhere in secure way. Biometric authentication uses real human physiological or behavioral characteristics to authenticate users. This proposed system uses voice recognition to authenticate every user before giving access to M-Commerce site by authenticating user on the basis of previously stored voice samples. The Proposed System will also support handicap people to get acess to sites being authenticated through voice without touching keyboard or mouse. This proposed system authentication try to remove limitation of sore throat and changing voice with time to some extent but not completely so it also includes in future work. One way is to provide other alternative to user like face recognition if user is suffering from sore throat .Future work also includes identify one's mental situation by identifying several acoustic features in the voice of speaker whether speaker is in depression, at high risk of suicide so in this way one can save one's life

\section{9 .ACKNOWLEDGMENTS}

Foremost, I would like to thank my family: my parents Mr. Vinay Kumar and Mrs. Shashi Bala for giving birth to me at the first place and supporting me spiritually throughout my life. Last but not the least; I would like to express my sincere gratitude to my advisor Mr. Sawal Tandon for the continuous support of my study and research, for his patience, motivation, enthusiasm, and immense knowledge. His guidance helped me in all the time of research

\section{REFERENCES}

[1] Gong Chen (2010) "Security System based on Speech Recognition in Mobile Commerce”,School Of Information Science and Engineering Southeast University Nanjing, China .

[2]Jiehua Dai, Zhengzhe Wei(2007) "Study and Implementation of Feature Extraction and Comparison in Voice Recognition".

[3]John Garofalakis,Flora (2008)"System's Design and implementation for easy creation of M-Commerce Systems",Flora Garofalakis,Vasilios Stefanis

[4]Mahmoud I. Abdalla and Hanaa S.Ali (2010) “WaveletBased Mel-Frequency Cepstral Coefficients for Speaker Identification using Hidden Markov Models". JOURNAL OF TELECOMMUNICATIONS, VOLUME 1, ISSUE 2, MARCH 2010.

[5]Qiong Long(2011) “A Construction Strategy Framework For M-Commerce Websites” Business School Beijing Institute of Fashion Technology Beijing, China

[6]Rajeev Aggarwal(2011)' Noise Reduction of Speech Signal using Wavelet Transform with Modified Universal Threshold". International Journal of Computer Applications (0975 - 8887) Volume 20- No.5, April 2011.

[7]Rashad Yazdanifard ,Mohamed Sayed (2011) “ $M$ Commerce and Related Mobile Security Issues", Faculty of Management, Multimedia University Technology, Cyberjaya, Malaysia.

[8]Yi Lei, Bingyong and Jin Lice(2011) “Evaluation of communication platform in E-Commerce and $M$ Commerce",Economic and management School NanChang Hangkong University Sponsor JiangXi Province Education and Science "12th five year plan"

[9]Jiehua Dai, Zhengzhe Wei(2007) “Study and Implementation of Feature Extraction and Comparison in Voice Recognition". A project report 